\title{
Advancement in Layered Transition Metal Dichalcogenide Composites for Lithium and Sodium Ion Batteries
}

\author{
Muhammad Yousaf, Asif Mahmood, Yunsong Wang, Yijun Chen, Zhimin Ma and Ray P. S. Han \\ Department of Materials Science and Engineering, College of Engineering, Peking University, Beijing 100871, China
}

\begin{abstract}
With an ever increasing energy demand and environmental issues, many state-of-the-art nanostructured electrode materials have been developed for energy storage devices and they include batteries, supercapacitors and fuel cells. Among these electrode materials, L-TMD (layered transition metal dichalcogenide) nanosheets (especially, S (sulfur) and Se (selenium) based dichalcogenides) have received a lot of attention due to their intriguing layered structure for enhanced electrochemical properties. L-TMD composites have recently been investigated not only as a main charge storage specie but also, as a substrate to hold the active specie. This review highlights the recent advancements in L-TMD composites with $0 \mathrm{D}$ (0-dimensional), 1D, 2D, 3D and various forms of carbon structures and their potential applications in LIB (lithium ion battery) and SIB (sodium ion battery).
\end{abstract}

Key words: L-TMD based composites, energy storage devices, nanocompsites, LIBs and SIBs.

\section{Introduction}

With the fast depletion of current energy sources on the pushed end, coupled with the increasing energy demands at the pulled end, it is not difficult to understand that the World will need new power sources of nearly $30 \mathrm{TW}\left(10^{12}\right)$ by 2050 [1]. This humongous new energy demand, together with the rising consumption of non-renewal fossil fuels (coal, oil, gas, etc.) and the difficult and often, conflicting environmental issues have created enormous pressures for the World to develop clean and sustainable technologies to rapidly provide for huge amounts of energy. It is necessary to develop renewable energy sources in order to fulfill the demands of modern society and eliminate environmental issues. In this regard, solar cells, fuel cell, wind and biomass-based energy are playing a vital role. To fully utilize these renewable energy resources, highly efficient, low cost, and environmental benignity energy storage systems

Corresponding author: Ray P. S. Han, Ph.D., professor, research fields: flexible electronic devices and nano/micro-fluidic devices. E-mail: ray-han@pku.edu.cn. are required. Among various energy storage systems, batteries (e.g. LIBs/SIBs) are attractive candidates for renewable energy storage due to their low cost, high energy density and environmental benignity [2-5]. Nevertheless, many challenges are still big hurdle in their real application due to lower power density and poor cyclic life. For example, most of electrode materials used in batteries have low conductivity, which affect the storage of ions and rate performance. In addition, large volume expansion during charging/discharging processes produces the strains in electrode materials, which can cause the delamination of active materials from current collector, resulted in severe capacity fading and poor cyclic stability. Besides, during volume expansion or in morphological and structural changes in most of electrode materials, causes the formation of newly SEI (solid electrolyte interface film), consequences the irreversible loss of capacity [2]. Therefore, electrode materials with high specific surface areas, high conductivity which could provide large sites for intercalation of ions for both insertion and conversion processes are required, 
eventually providing opportunities to tailor high energy density and power density devices for practical applications.

In recent years, 2D layered materials have attracted enormous attention due to their unique physical, chemical and electronic properties [6-8]. Among various layered structures, graphene with its excellent properties (conductivity, large surface area, flexibility, and the ability to functionalize with other molecules) is probably the most popular as it possesses great potentials in Li-based batteries, supercapacitors, photovoltaic devices, fuel cells etc. [9-15]. With motivation from graphene, other layered inorganic nanomaterials, particularly L-TMD (layered transition metal dichalcogenides) such as $\mathrm{MoS}_{2}, \mathrm{MoSe}_{2}, \mathrm{WS}_{2}$, $\mathrm{SnS}_{2}, \mathrm{SnSe}_{2}$, etc. have also received wide attention from scientific communities [16-20]. They are compounds with the formula $\mathrm{MX}_{2}$, where $\mathrm{M}$ is a transition metal element of group IV-B (Ti, Zr, Hf, etc.), $\mathrm{V}-\mathrm{B}(\mathrm{V}, \mathrm{Nb}, \mathrm{Ta}$, etc.), VI-B (Mo, W, Sn, etc.), and $\mathrm{X}$ is a chalcogen of the VI-A group ( $\mathrm{S}, \mathrm{Se}$, etc.). In these materials, the interlayer atoms are strongly held together by covalent bonds, while the individual layers are weakly held in place by the van der Waal interaction to form a sandwich-like X-M-X structure, [21-23] where the $M$ atom layer is sandwiched between the two X layers. These compounds have crystalline structure that varies from the hexagonal $\left(\mathrm{MoS}_{2}\right.$ and $\mathrm{WS}_{2}$ ) to the orthorhombic $\left(\mathrm{MoTe}_{2}\right.$ and $\left.\mathrm{WTe}_{2}\right)$. Such compounds are very important in industry and from scientific point of view, they are interesting. For instance, owing to their layered structure, $\mathrm{MoS}_{2}$ and $\mathrm{WS}_{2}$ have been utilized as dry lubricants in industry as they can withstand higher temperatures than graphite [24, 25]. In addition, L-TMD offers unique electronic, physical or chemical properties compared to its bulk counterparts and this makes it attractive for various applications [26, 27]. Moreover, due to their higher surface area and being active electronically and chemically, these 2D nanomaterials have been used in electronic/optoelectronic devices, sensors, electrocatalysis, energy storage devices and many other applications $[28,29]$. However, there are some challenges associated with these L-TMD NSs (nanosheets) in energy storage due to their inherent limitations [30]. For example, the low electronic conductivity of L-TMD NSs will affect their electrochemical performance, and they also suffer from large mechanical stress and volume change during charging/discharging, which can induce pulverization and aggregation of active electrode materials, resulting in poor cyclic stability.

To overcome the weakness of individual counterparts, the hybridization of two or more nanomaterials is an intriguing approach to engineer target materials with multifunctionalties, and improved properties for practical applications [31,32]. Similar to 2D graphene, L-TMD NSs have also been incorporated into a number of materials to improve their electrochemical properties. With an ever-increasing demand for energy storage devices and increasing interest in L-TMD from the scientific community, we aim to give a mini-review of this immensely important and rapidly growing research field. First, we will elaborate various approaches for the preparation of L-TMD nanocomposites with $0 \mathrm{D}, 1 \mathrm{D}, 2 \mathrm{D}, 3 \mathrm{D}$ and others as shown schematically in Fig. 1. Then, we will highlight the promising application of these L-TMD composites in LIBs and SIBs. Hopefully, this article

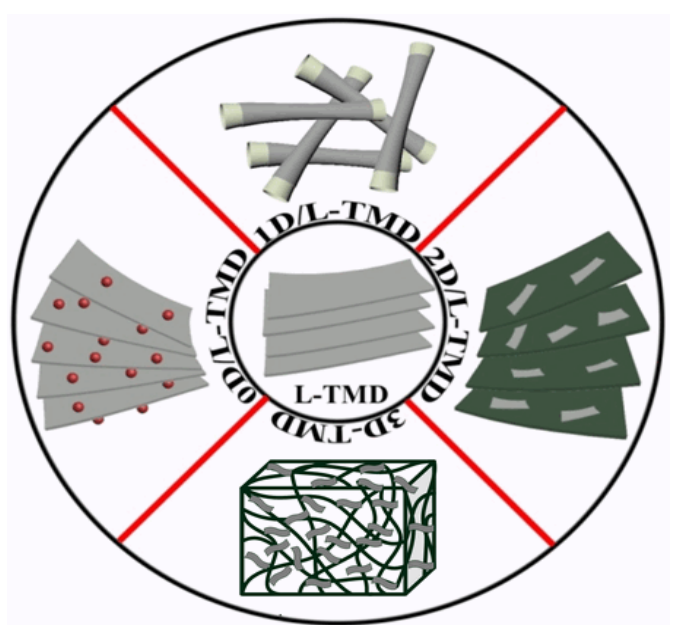

Fig. 1 Schematic illustration of L-TMD composites with 0D, 1D, 2D, 3D. 
will be beneficial to researchers and materials scientists.

\section{Different Approaches to Prepare L-TMD-Based Composites}

The charge storage capabilities of the electrode materials depend strongly on the exposed surface of the active materials. Owing to largely exposed external surface of TMD layers, the L-TMDs present unique materials (similar to graphene in some extent) with high surface area, which benefits from their ultrathin sheets and 2D morphology. However, the adjacent 2D layers can be easily restacked together under van der Wall interaction during cycling processes in batteries that resulted in a loss of the 2D structure and significant decreases of their active surface area. Hybridization of L-TMD with other functional material is an effective strategy to retain their inherent structure and hence, the inherent specific surface area for improved electrochemical response. Recently, there have been a lot of developments on the hybridization of L-TMD NSs with 0D (NPs (Nanoparticles)), 1D, 2D and 3D structures.

\subsection{Composites of L-TMD with OD (NPs) Structures}

The electrochemical applications of L-TMD are undermined by poor conductivity and restacking (limiting effective surface area) during the reversible charge storage. A possible way to tailor the L-TMD materials with enhanced conductivity and good control over the surface area during cycling is to incorporate the 0D moieties (noble metal, and metal/metal oxide (NPs) etc.) in the layered structures which can provide charge carriers for enhanced conductivity and effectively limit the restacking of L-TMDs [33-38]. Such incorporation acts as a spacer to support the material, making the active surfaces of L-TMD accessible for electrolyte penetration lithiation/de-lithiation process improving electrochemical response of the LIBs. Recently, the incorporation of noble metals ( $\mathrm{Au}, \mathrm{Ag}, \mathrm{Pt}, \mathrm{Pd})$ [38-41] has been identified as a possible solution to improve the inherent conductivity of L-TMDs. For instance, Pan et al. [38] anchored the Ag NPs on $\mathrm{MoS}_{2} \mathrm{NSs}$ through coordination by using multifunctional organic ligand. Ag NPs not only improve the conductivity (due to the higher intrinsic conductivity of noble metals) but also, act as a spacer and inhibit the restacking or agglomeration of $\mathrm{MoS}_{2}$. Further, due to the high inflexibility and low deformability of $\mathrm{Ag}$ NPs effectively preserve the composite structure [38]. Similarly, Chen et al. used ultrasmall $(\sim 3.5 \mathrm{~nm}) \mathrm{Fe}_{3} \mathrm{O}_{4}$ NPs to decorate the $\mathrm{MoS}_{2}$ NSs by a 2-part hydrothermal process. Authors argue that due to elastic and flexible nature, the $\mathrm{MoS}_{2} \mathrm{NSs}$ are more active electrochemically than reduced graphene oxide (rGO) and act as a better substrate for the growth of $\mathrm{Fe}_{3} \mathrm{O}_{4}$ NPs and accommodate the strain. The ultrasmall $\mathrm{Fe}_{3} \mathrm{O}_{4}$ NPs also act as spacer between the sheets of $\mathrm{MoS}_{2}$ and prevent the composites from collapsing during charging and discharging in an LIB, where as the L-TMDs without spacer tended to crumpled and agglomerate (Figs. 2a and 2b) [33], severely limiting the reversible capacity and cycling performance of the LIB. In similar attempts, metal NPs have also been decorated on $\mathrm{MoS}_{2}$ NSs. For instance, Sn NPs were embedded in layered $\mathrm{MoS}_{2}$ by two step hydrothermal method. The $\mathrm{MoS}_{2}-\mathrm{C}$ hybrids were fabricated from $\mathrm{Na}_{2} \mathrm{MoO}_{4} \cdot 2 \mathrm{H}_{2} \mathrm{O}, \mathrm{NH}_{2} \mathrm{CSNH}_{2}$ and glucose as source material by hydrothermal method with subsequent heat treatment in Ar atmosphere. The resultant product was then subjected to another hydrothermal reaction in presence of Sn precursor followed by final calcination in $\operatorname{Ar}$ [37].

Keeping in mind the individual advantages of noble metals and other metal nanostructures, the co-doping of noble metal along with electrochemical active metal nanostructures could effectively provide dual advantage of better conductivity and reversible capacity [34]. Recently, Pan et al. proposed the development of ternary heterostructures $\left(\mathrm{Ag} / \mathrm{Fe}_{3} \mathrm{O}_{4} / \mathrm{MoS}_{2}\right)$ by one step, in which as prepared 


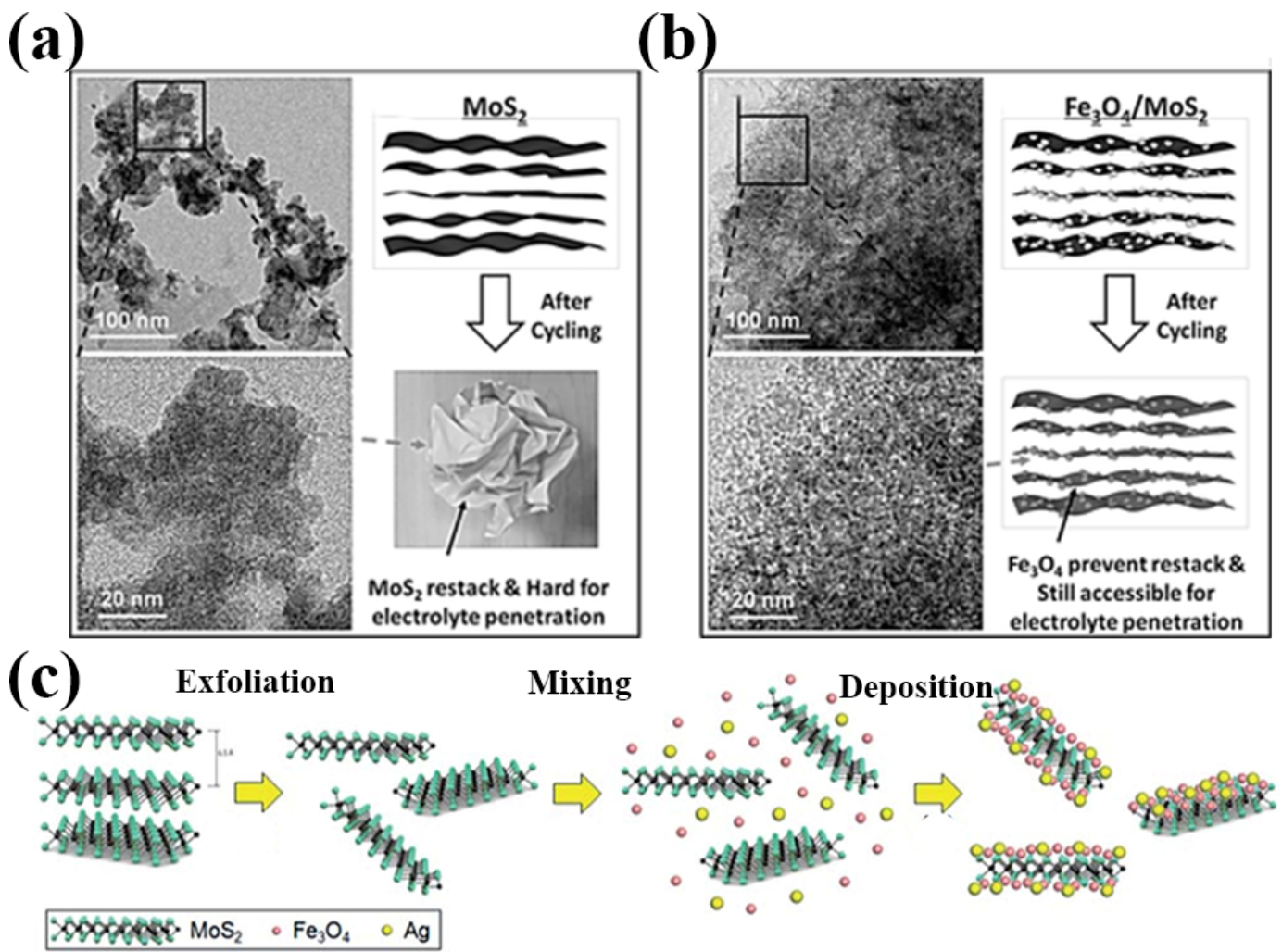

Fig. 2 (a) TEM images and schematic illustration of pristine $\mathrm{MoS}_{2}$; (b) $\mathrm{Fe}_{3} \mathrm{O}_{4} / \mathrm{MoS}_{2}$ before and after 1180 cycles of charge/discharge [33]. (a, b) Reproduced with permission of John WILEY \& Sons, Inc.; (c) Schematic illustration of Exfoliation of $\mathrm{MoS}_{2}$ powder into NSs, mixing of exfoliated $\mathrm{MoS}_{2}$ with $\mathrm{Fe}_{3} \mathrm{O}_{4}$ and $\mathrm{Ag} \mathrm{NPs}$ and deposition of NPs on MoS $\mathrm{NSs}$ through van der Waals interactions [34]. Reproduced with permission of The Royal Society of Chemistry.

$\mathrm{Fe}_{3} \mathrm{O}_{4}, \mathrm{Ag}$ NPs and exfoliated $\mathrm{MoS}_{2}$ NSs were mixed in THF (tetrahydrofuran) by magnetic stirring and these NPs were spontaneously decorated onto naked surfaces of $\mathrm{MoS}_{2}$ by van der Waals interactions (Fig. 2c) [34]. Here, Ag NPs enhance the intrinsic conductivity of composites while $\mathrm{Fe}_{3} \mathrm{O}_{4}$ stores lithium reversibly. Despite considerable efforts, it is a big challenge to obtain single layer as it is difficult to control the layer number by hydrothermal process and could be a topic for future research as it will not only provide the insertion and conversion sites for lithium storage, but also numerous interfacial Li storage sites.

\subsection{Composites of L-TMD with 1D Structure}

1D nanostructures have also been used to hybridize with L-TMD NSs to improve the reversible charge storage of electrode materials. As an attractive 1D nanostructure, single wall and/or MWCNT (multiwalled carbon nanotubes) have received a great deal of attention due to their excellent mechanical and electronic properties. The 1D nature of the CNTs provides pathways for conduction during cycling, which make them excellent substrates for the growth of L-TMD NSs for Li storage [42-46]. Further, due to their high flexibility, CNTs also help to accommodate volume expansion of electrode materials during charging/discharging. Several efforts have been made to tailor efficient $1 \mathrm{D}$ electrode materials using CNTs as seed to grow L-TMD NSs. Ding et al. [42] used a simple hydrothermal approach for the growth of $\mathrm{MoS}_{2}$ NSs on the surface of CNT from sodium molybdate hexahydrate and thiourea precursor in the presence of glucose. The synthesis method not only ensured homogeneous growth of $\mathrm{MoS}_{2}$ on CNT surface but also, a good conductive network formed from pyrolysis of glucose, which ensured the excellent contact between CNT backbone and $\mathrm{MoS}_{2}$ NSs. Recently, many nanostructured materials have been prepared by using 
biomolecular-assisted synthetic approaches [43, 47].

Among numerous biomolecules, L-cys (L-cysteine) is very promising due to its special structure, which contains many functional groups $\left(-\mathrm{NH}_{2},-\mathrm{SH}\right.$, and - $\mathrm{COO}$ ) and has been utilized as sulfur source and reductant in hydrothermal synthesis approaches. A simple hydrothermal method was used to synthesize $\mathrm{CNTs}-\mathrm{MoS}_{2}$ hybrid by reaction between sodium molybdate dehydrate and L-cys in the presence of acid treated CNTs with subsequent annealing treatment [43]. The electrostatic interaction between the negatively charged MWCNT and $\mathrm{Sn}^{4+}$ ion played a critical role for the decoration of $\mathrm{SnS}_{2}$ NSs on MWCNT, followed by the nucleation and 2D growth of $\mathrm{SnS}_{2}$ as shown in Fig. 3a. Besides, TMD-coated hybrid nanocomposites, single- or multi-layered $\mathrm{MoS}_{2}$-embeded carbon nanotube/nanofibers or nitrogen doped CNF (carbon nanofibers) have also been reported [48-50]. Kong et al. [49] fabricated $\mathrm{MoS}_{2}$ NSs embedded-graphitic nanotubes structure in which graphene rolled up into hollow nanotubes and thin $\mathrm{MoS}_{2}$ NSs were standing on the inner surface of graphitic carbon. Recently, the electrospinning method has been identified as an excellent way to obtain single layers of L-TMD NSs and this is demonstrated by the electrospun single layers of $\mathrm{WS}_{2}$ nanoplates that were homogeneously embedded in NCNFs (nitrogen-doped carbon nanofibers) [50].

Additionally, L-TMD NSs have also been decorated on the non-carbon based 1D structure. For example, several layers of $\mathrm{MoS}_{2} \mathrm{NSs}$ were decorated on $\mathrm{TiO}_{2}$ nanotubes/nanowires/nanobelts by the hydrothermal/solvothermal approach [51-53]. Li et al. fabricated a hierarchical nanocomposite, in which $\mathrm{MoS}_{2}$ NSs were coated on $1 \mathrm{D} \mathrm{TiO}_{2}$ nanowires by a cost-effective glucose-assisted hydrothermal route as illustrated in Fig. 3b. The robust nature of $\mathrm{TiO}_{2}$ served as the effective template for the growth and nucleation of the layered-MoS $\mathrm{MSs}_{2}$. Both the roughness of $\mathrm{TiO}_{2}$ and glucose played an important role in homogenous coating of $\mathrm{MoS}_{2} \mathrm{NSs}$ in the composite [52]. However,

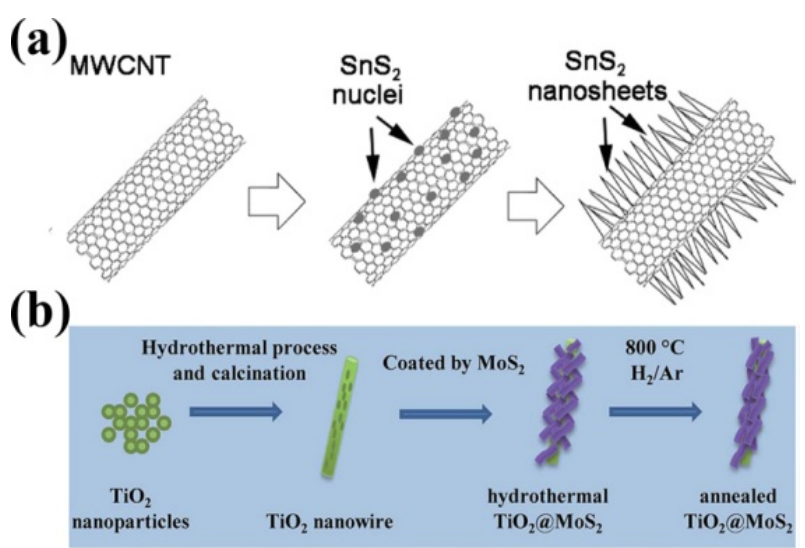

Fig. 3 (a) Schematic representation for the synthesis process of the $\mathrm{SnS}_{2}$ NS@MWCNTs coaxial nanocables [45]. Reproduced with permission of American Chemical Society; (b) Schematic description of the growth process of the $\mathrm{TiO}_{2} @ \mathrm{MoS}_{2}$ nanocomposite [52]. Reproduced with permission of The Royal Society of Chemistry.

uniform and controllable deposition of $\mathrm{MoS}_{2}$ on $\mathrm{TiO}_{2}$ surface is still a big challenge due to its poor affinities and chemical reactions.

\subsection{Composites of L-TMD with 2D Structure}

The excellent properties of L-TMDs have been further exploited by growing them on flexible $2 \mathrm{D}$ substrates. Graphene constitutes an excellent substrate material with exceptional electrochemical properties due to the extended $\pi$-conjugation and honey comb-like structure. It is also the most conductive form of carbon with excellent mechanical properties and good flexibility spread over a large surface area. Incorporation of graphene with L-TMD NSs can enhance the electrochemical performance of L-TMD by improving the (i) electrochemical surface area; (ii) electrode/electrolyte interface; (iii) inherent conductivity by providing pathways for electronic conduction; (iv) ease of mass diffusion, etc. Surfactant assisted hydrothermal/wet chemical method is the most common approach for the preparation of single- or multi-layered $\quad \mathrm{L}_{-} \mathrm{MoX}_{2} \quad(\mathrm{X}=\mathrm{S}, \quad \mathrm{Se})$-graphene composites [47, 54-60]. For instance, Chang et al. presented a novel approach for the growth of layered $\mathrm{MoS}_{2} /$ graphene hybrids by an L-cys-assisted solution-phase technique; using sodium molybdate, 
L-cys and GO (graphene oxide) as a precursor with a subsequent heat treatment [47]. Similarly, wet-chemical routes have used to synthesize single layered $\mathrm{MoS}_{2} / \mathrm{GNS}$ hybrid materials by CTAB (cetyltrimethylammonium bromide) assisted with a reduction of $\left(\mathrm{NH}_{4}\right)_{2} \mathrm{MoS}_{4}$ and $\mathrm{GO}$ sheets by hydrazine, followed by a heat treatment in an $\mathrm{N}_{2}$ atmosphere [56]. Other cationic surfactants such as dodecyltrimethylammonium bromide, DTAB; octocyltrimethy-lammonium bromide, OTAB; tetrabutylammonium bromide TBAB have also been used for the synthesis of layered $\mathrm{MoS}_{2} / \mathrm{GNS}$ hybrids $[57,58]$. The use of surfactant helps to control the morphology of the resulting product, which in turn results in a better control of the performance of the hybrid materials. In addition, the use of different cationic surfactants and their concentration in synthesis can be used for controlling the number of layers of $\mathrm{MoS}_{2}$ [57].

Recently, NG (nitrogen doped graphene) has received greater attention as a suitable substrate for the growth of nanomaterials for nanostructured electrodes due to their high roughness and wrinkled surface with large number of defect sites and vacancies. Chang et al. developed $\mathrm{MoS}_{2} / \mathrm{NG}$ composite by a facile two step mechanism where L-cys was used as S source and doped in $\mathrm{NG}$ by heating at an elevated temperature followed by in-situ $\mathrm{MoS}_{2} \mathrm{NSs}$, as depicted in Fig. 4a [61]. Besides wet chemical/hydrothermal routes, a scalable liquid phase exfoliation approach was used to

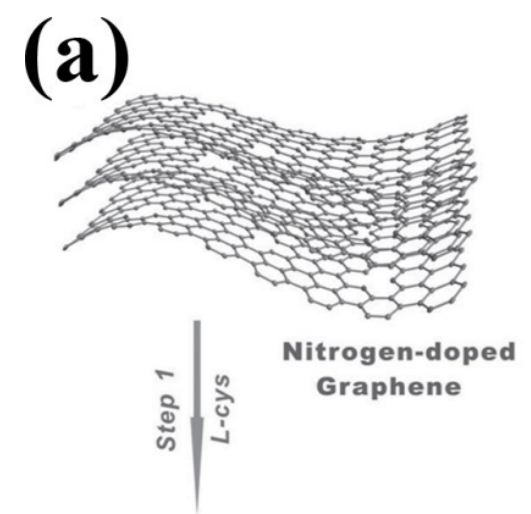

\section{(b)}
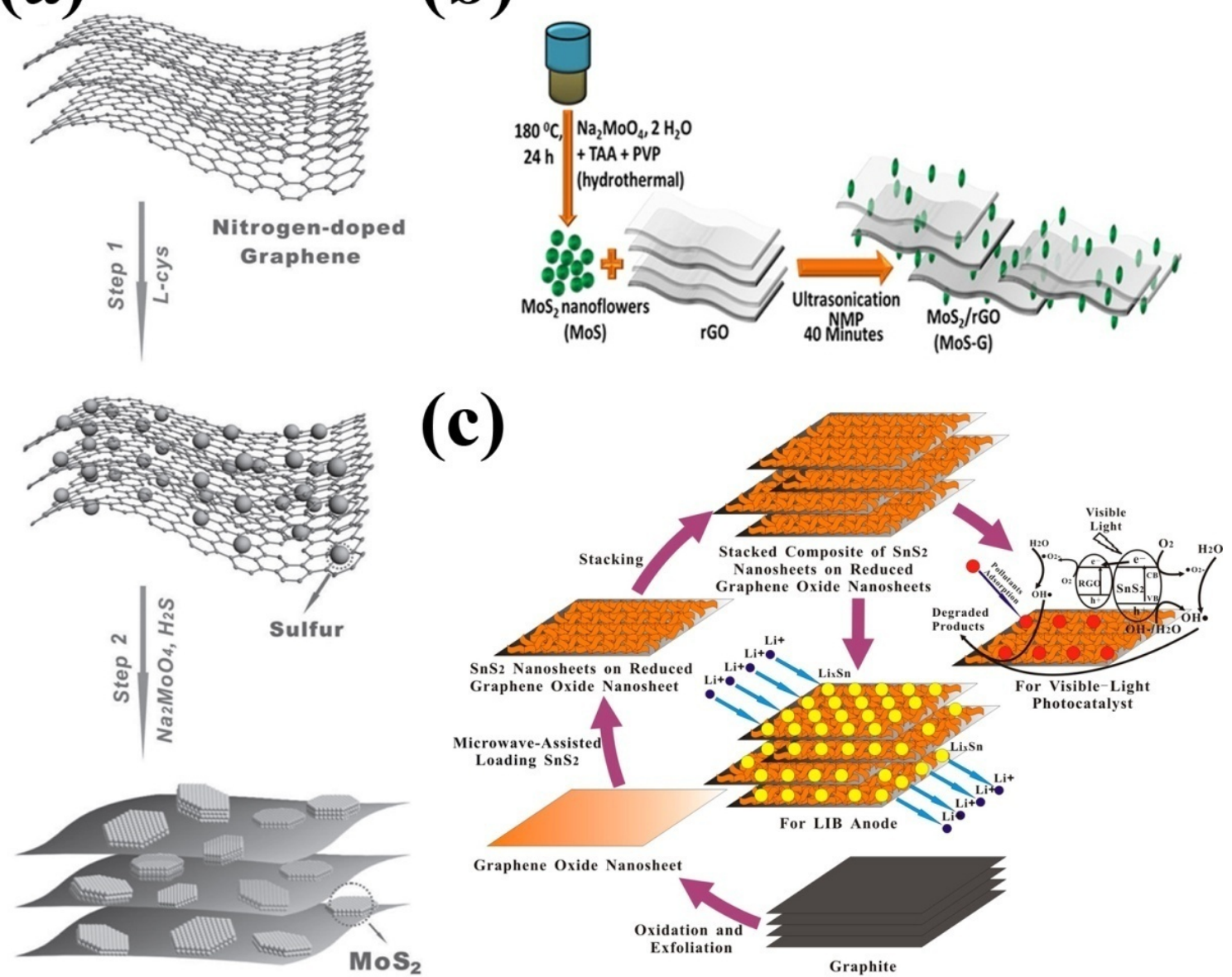

Fig. 4 (a) Schematic representation of synthesis process of $\mathrm{MoS}_{2} / \mathrm{NG}$ composite [61]. Reproduced with permission of John WILEY \& Sons, Inc.; (b) Schematic representation of $\mathrm{MoS}_{2}$ nanoflowers (MoS) and $\mathrm{MoS}_{2} / \mathbf{r G O}$ composite (MoSG) [62]. Reproduced with permission of Nature Publishing Group; (c) Schematic description of the growth mechanism and applications of $\mathrm{SnS}_{2}$-RGO sheet-on-sheet nanostructure [64]. Reproduced with permission of American Chemical Society. 
synthesize $\mathrm{MoS}_{2}$-rGO composite, in which the PVP assisted $\mathrm{MoS}_{2}$ NSs were first prepared by a low temperature hydrothermal method and the flower like $\mathrm{MoS}_{2}-\mathrm{rGO}$ composite prepared by ultrasonic exfoliation technique (Fig. 4b) [62]. Further, a layered by layered $\mathrm{MoS}_{2} / \mathrm{rGO}$ composite was synthesized via chemical lithiation and exfoliation methods with less stacked $\mathrm{MoS}_{2}$ layers with 1 3 layers [63].

Other L-TMDs such as $\mathrm{SnX}_{2}(\mathrm{X}=\mathrm{S}, \mathrm{Se})$ materials have also been composited with graphene to enhance their electrochemical performance. Similar to $\mathrm{MoX}_{2}$, wet-chemical-methods have also been most common approaches to synthesize nanocomposites of $\mathrm{SnX}_{2}$ with graphene [65-69]. For example, L-cys assisted FL-SnS $2 / \mathrm{G}$ (few-layer $\mathrm{SnS}_{2} /$ graphene) composites were synthesized by solution-chemistry method, in which L-cys was used as sulfur source, complexing and reducing agent [66]. Another technique was also used to fabricate sheet-on-sheet $\mathrm{SnS}_{2} / \mathrm{RGO}$ via a 1-step microwave assisted method for enhanced electrochemical performance and photocatalytic applications, as shown in Fig. 4c [64]. A CVD approach has also been used for $\mathrm{SnS}_{2}$-graphene composites. The 2-step approach was used to synthesize 2D graphene- $\mathrm{SnS}_{2}$ composites, with the $\mathrm{SnO}_{2} \mathrm{NPs}$ anchored in the graphene NSs and then, converted into the metal disulfide nanoplates via a solution method followed by a simple CVD process [70]. In addition, graphene supported $\mathrm{WS}_{2}$ nanocomposites have also been synthesized and investigated to improve the electrochemical performance of $\mathrm{WS}_{2}$ because of its higher conductivity than $\mathrm{MoS}_{2}$ [71-74]. A solution-phase method was reported for the synthesis of few-layer $\mathrm{WS}_{2}$ supported on RGO (reduced graphene oxide) by reduction of GOS dispersion with hydrazine hydrate under reflux [71].

\subsection{D Porous Structures with 2D Graphene/Porous CNF}

Although the nanocomposites of L-TMD with graphene showed much better performance, but these L-TMD NSs are affected by strong aggregation upon successive cycling because of non intimate contact between graphene layers and L-TMD, and later suffers from large contact resistance among numerous active NSs. Recently, 3D porous architecture of $\mathrm{MoX}_{2}(\mathrm{X}=\mathrm{S}$, $\mathrm{Se})$ with graphene and porous $\mathrm{CNF}$ have been reported [75-79]. For instance, a bottom-up approach is developed to synthesize with 3D porous architecture from exfoliated $\mathrm{MoS}_{2}$-GNS ( $\mathrm{MoS}_{2} \mathrm{NSs}$ and GO NSs) as building block and afterwards hydrothermal reaction (Fig. 5a) [75] in which $\mathrm{MoS}_{2}$ NSs were confined in the matrix of graphene as shown in Fig. 5b. Recently, both graphene and o-MWCNT (oxidized MWCN) together combined with $\mathrm{MoS}_{2}$, to synthesize the 3D f-MoS (flower-like $\mathrm{MoS}_{2}$ ) hierarchical structure (f- $\mathrm{MoS}_{2} / \mathrm{RGO} / \mathrm{o}-\mathrm{MWCNT}$ ) [77]. Addition of o-MWCNT in the composites resulted in the formation of flower-like morphology and also inhibited the stacking of RGO. Besides $\mathrm{MoS}_{2}$, first time 3D mesoporous $\mathrm{MoSe}_{2} / \mathrm{rGO}$ foam was synthesized by hydrothermal method, which was used as anode in LIB [78]. Additionally, L-TMD NSs such as the $\mathrm{SnS}_{2}$ and $\mathrm{WS}_{2}$ have also been combined with the $3 \mathrm{D}$ porous structure to improve their electrochemical performance [80-84]. Recently, a facile two-step route to synthesize 3D interconnected graphene frameworks embedded with homogeneously distributed $\mathrm{SnS}_{2}$ nanoplates (SSG) by using GO NSs, $\mathrm{SnCl}_{4} .5 \mathrm{H}_{2} \mathrm{O}$ and L-cys as source materials has reported. Firstly $\mathrm{SnO}_{2}$ NPs decorated on GO NSs via water bath strategy [85] and then directly transformed into $\mathrm{SnS}_{2}$ by hydrothermal treatment with L-cys as reducing agent and sulfur source [81]. Additionally, Huang et al. [82] reported a layered composite $\mathrm{WS}_{2}$ with $3 \mathrm{D}$-graphene interconnected networks by hydrothermal method at relatively low temperature for LIB and hydrogen evolution reactions.

Besides the 3D architectures of $\mathrm{MoS}_{2}$ with carbonaceous materials, recently $3 \mathrm{D}$ networks of $\mathrm{MoS}_{2}$ have also been prepared with $\mathrm{TiO}_{2}$ microspheres to 
enhance the electrochemical performance of LIBs [86]. For instance, a firework shaped $\mathrm{TiO}_{2}\left(3 \mathrm{D}\right.$ F-TiO $\left.{ }_{2}\right)$ microspheres have been used as template, in which $3 \mathrm{D}$ $\mathrm{F}-\mathrm{TiO}_{2}$ microspheres were embedded with $\mathrm{MoS}_{2} \mathrm{NSs}$ by two step hydrothermal methods [86].

\subsubsection{Composites of L-TMD with 3D Bulk Materials}

In addition to developing 3D architectures from 2D graphene, 3D graphene has itself been employed to tailor the electrode materials. CVD is used successfully to grow 3D graphene network (3DGNs) which is used as substrate to grow nanomaterials resulting in functional composites [87, 88]. The 3DGNs not only offer high surface area, but also preserve the excellent conductivity of graphene. In a synthesis strategy, $\mathrm{MoS}_{2}$ flakes were coated on 3DGNs through CVD using $\left(\mathrm{NH}_{4}\right)_{2} \mathrm{MoS}_{4}$ dissolved in DMF (dimethylformamide) as a precursor [89]. Similarly, $\mathrm{MoS}_{2}$ NSs can also be deposited on ACF (activated carbon fiber) cloth. Wang et al. [90] reported a two step method to grow ultrathin $\mathrm{MoS}_{2}$ NSs on the surface of ACF cloth using $\left(\mathrm{NH}_{4}\right)_{2} \mathrm{MoS}_{4}$ dissolved in DMF (dimethylformamide) as source material in the presence of pre-annealed ACF cloth with subsequent annealing treatment in $5 \% \mathrm{H}_{2} / \mathrm{Ar}$ atmosphere. The formation of $\mathrm{MoS}_{2}$ is described by the following equation [91].

$$
\left(\mathrm{NH}_{4}\right)_{2} \mathrm{MoS}_{4}+\mathrm{H}_{2} \rightarrow 2 \mathrm{NH}_{3} \uparrow+2 \mathrm{H}_{2} \mathrm{~S} \uparrow+\mathrm{MoS}_{2}
$$

\subsection{Composites of L-TMD with Various Other Forms of} Carbon

The amorphous form of carbon has also been used for templates and matrices for growing the L-TMD [92-98]. For example, Chang et al. [92] developed $\mathrm{MoS}_{2} /$ amorphous carbon composites using simple hydrothermal process followed by high temperature calcination. Similarly a facile and simple solution-phase method was used to synthesize the sheet-like $\mathrm{MoSe}_{2} / \mathrm{C}$ composite from $\mathrm{Na}_{2} \mathrm{MoO}_{4} \cdot 2 \mathrm{H}_{2} \mathrm{O}$, Se powder and glucose as the precursor and following with heat treatment. The carbon in the composite maintains structural integrity and also inhibits the stacking of NSs [96]. Recently, different carbon hollow

structures such as nitrogen doped hollow carbon nanobox and PHCS (porous hollow carbon spheres) have also been used for the growth of L-TMD NSs. These hollow carbon structures increase the conductivity of nanocomposites and also inhibit the aggregations of L-TMD NSs during cycling [94, 95]. The composite of N-doped carbon nanobox with $\mathrm{MoS}_{2}$ mainly involve 4 steps as shown in Fig. 5c. This carbon shell greatly improved the conductivity of the composite, accommodated the strain and effectively prevented the aggregation of $\mathrm{MoS}_{2}$ NSs during cycling processes.

\section{(a)}
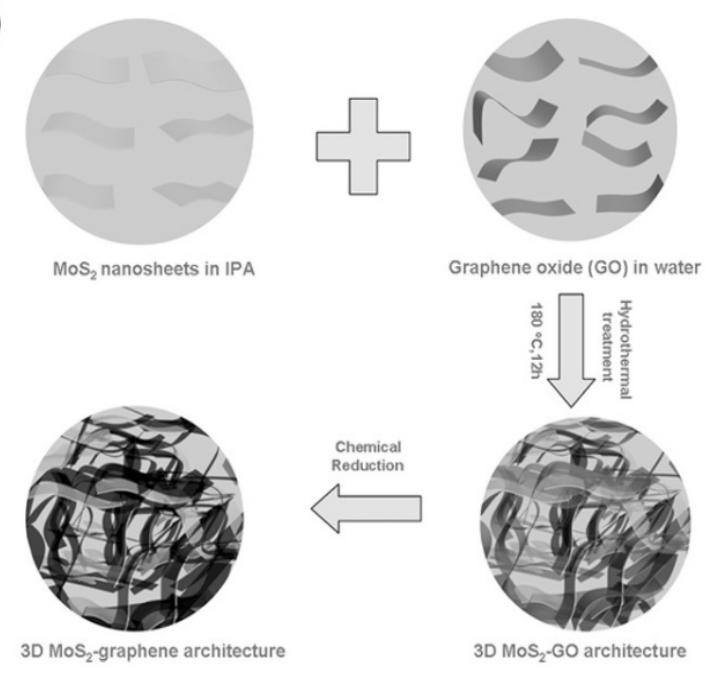

(b)
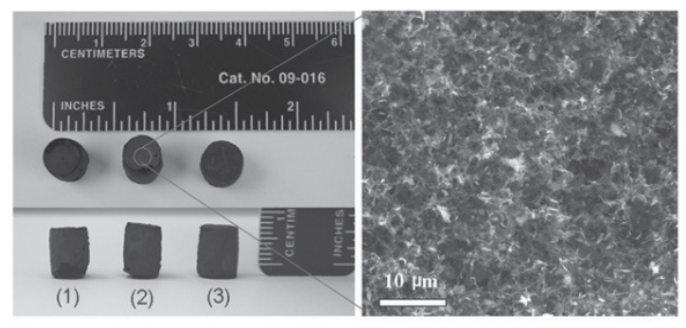

(c)

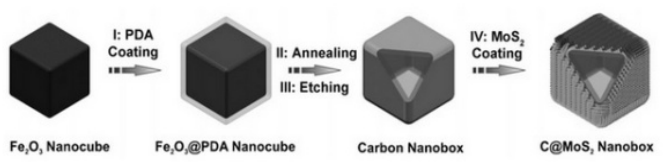

Fig. 5 (a) Schematic representation for the growth mechanism of 3D $\mathrm{MoS}_{2}$-graphene architectures; (b) Photographs and SEM of 3D $\mathrm{MoS}_{2}$-graphene architectures, respectively [75]. (a, b) Reproduced with permission of WILEY \& Sons, Inc. (c) Schematic representation of synthesis process for $\mathrm{C} @ \mathrm{MoS}_{2}$ nanobox [94]. Reproduced with permission of WILEY \& Sons, Inc. 


\section{Prospective Applications of L-TMD Composites}

With an ever increasing energy demand and resource depletion crisis, the storage of green and sustainable energy has become a major challenge for materials scientists and researchers. In recent years many new energy materials have been synthesized to meet these challenges. Due to improved physical and electrochemical properties, L-TMD nanocomposites appear to lead the way for many advanced applications in the development of energy storage devices. In this section, we will briefly review the potential applications of the nanocomposites for high performance LIB and SIB batteries.

\subsection{LIBs (Lithium Ion Batteries)}

Rechargeable LIBs are now used extensively as a power source for portable electronic devices (such as laptops, notebooks, mobile phones, etc.), EVs (electric vehicles) and HEVs (hybrid electric vehicles) and this can be attributed to the many merits of LIBs; the principal ones are: high energy density, durable cycle life and no memory effect [2, 5, 99]. As an anode material in LIBs, graphite has been widely employed; however, due to its low specific capacity $\left(372 \mathrm{mAhg}^{-1}\right)$ and relatively poor rate capability, it cannot fulfill the demand of EVs and HEVs. L-TMD NSs have relatively high specific capacity compared to graphite, shortened path lengths for $\mathrm{Li}^{+}$ion and large exposed surface area, which plays an important role in enhancing the performance of LIBs. However, they have poor cycle life and rate capability as a result of the low intrinsic conductivity and easy restacking during $\mathrm{Li}^{+}$insertion/de-insertion [100]. The hybridization of L-TMD with different morphologies such as $0 \mathrm{D}$ (noble metals, metal/oxides NPs), 1D (CNT,CNF, $\mathrm{TiO}_{2}$ nanotubes/nanowires/nanobelts), 2D (graphene, $\mathrm{TiO}_{2}$ nanosheets), 3D (3D graphene, ACF, 3D porous architectures) and other forms of substrates is one of the most promising approaches to enhance the reversible capacity, cycle ability and rate performance of LIBs.

In this regard, composites of L-TMD NSs with 2D graphene are the most studied nanomaterials for LIBs [47, 54-60, 63-72, 74]. As a typical example, layered $\mathrm{MoS}_{2} / \mathrm{G}$ ( $\mathrm{MoS}_{2} /$ graphene) composite was reported by solution-phase approach, when this composite was used as anode in LIBs, it exhibited a high specific capacity of about $1,100 \mathrm{mAhg}^{-1}$ at $0.1 \mathrm{Ag}^{-1}$ current density with an excellent cycling stability (100 cycles). It also showed good rate capability with the specific capacity at about $900 \mathrm{mAhg}^{-1}$, even at $1.0 \mathrm{Ag}^{-1}$ current density [47]. The enhanced electrochemical performance of the composites was attributed to the synergistic effect between L-TMD and GNS. GNS not only preserved the electronic conductivity of the composites, but it also, reduced the contact resistance of the electrode. Further, the flexible nature of GNS absorbed the stress peaks induced during the cycling processes. In addition, many composites of $\mathrm{WS}_{2}, \mathrm{SnS}_{2}$, and $\mathrm{SnSe}_{2}$ with graphene for the improved performance of LIBs have been reported [64-72, 74]. Doping is another strategy used to further increase the conductivity of graphene, which resulted in high lithium storage capacity. N-G (nitrogen-doped graphene), the more conductive form of GNS has also been used for synthesizing $\mathrm{MS}_{2}(\mathrm{M}=\mathrm{Mo}, \mathrm{W}) / \mathrm{N}-\mathrm{GNS}$ composites [61, 73]. Due to N-doping, the defect sites and vacancies for $\mathrm{Li}^{+}$ions active sites extend gradually during cycling process and resulted in high performance in terms of capacity and cyclic stability. (Fig. 6a) [61]. For example, $\mathrm{MoS}_{2} / \mathrm{N}-\mathrm{G}$ composite possesses better reversible capacity than $\mathrm{MoS}_{2}$, $\mathrm{MoS}_{2} / \mathrm{G}$ and good rate capability for LIBs as shown in Figs. $6 \mathrm{~b}$ and $6 \mathrm{c}$ which clearly demonstrated that defect sites or vacancies are gradually extending and facilitating more $\mathrm{Li}^{+}$ions for insertion in N-G structure during charging/discharging.

3D porous interconnected architectures of L-TMD with varying carbon structures of $2 \mathrm{D}$ graphene and porous CNF have been used as an anode for LIBs [75-82, 84]. These porous structures not only provide a 
(a)

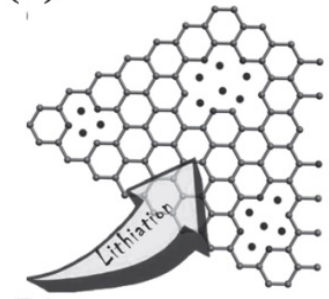

(b)

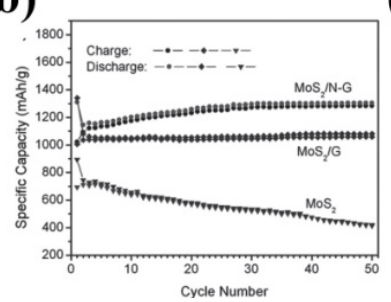

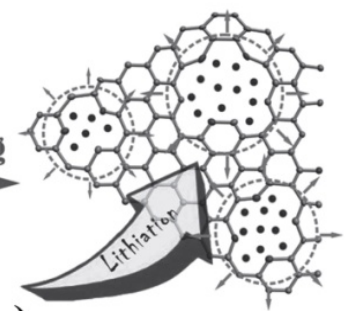

(c)

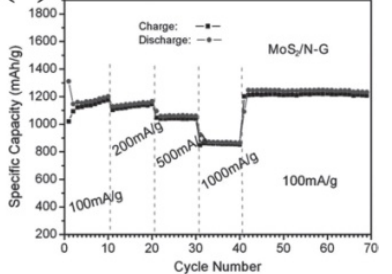

Fig. 6 (a) Schematic description of N-G during cycling: the defect sites or vacancies are extending gradually, facilitating more intercalation of $\mathrm{Li}^{+}$ions, blue balls represent $\mathrm{Li}^{+}$ions; (b) Cycling behaviors of $\mathrm{MoS}_{2}, \mathrm{MoS}_{2} / \mathrm{G}$ and $\mathrm{MoS}_{2} / \mathrm{N}-\mathrm{G}$ samples at a current density of $0.1 \mathrm{mAhg}^{-1}$; (c) Cycling performance of $\mathrm{MoS}_{2} / \mathrm{N}-\mathrm{G}$ electrode with different current densities [61]. Reproduced with permission of John WILEY \& Sons, Inc.

larger surface area, increased channels for easy electrolyte access and continuous pathways for rapid charge transfer, but they also, provide a cushion for the volume expansion of L-TMD nanosheets during the charging/discharging. As a result, the high specific capacity, excellent cyclic performance and ultrahigh-rate capability are achieved using 3D porous interconnected architectures. For instance, 3D porous graphene architecture coated with $\mathrm{MoS}_{2}$ by the hydrothermal method for high performance of LIB has been reported with improved capacity retention $(1,200$ $\mathrm{mAhg}^{-1}$ after 3,000 cycles), and excellent rate capability with capacity of $270 \mathrm{mAhg}^{-1}$ at $140 \mathrm{C}$ [76]. Also, the CVD method is used to deposit $\mathrm{MoS}_{2}$ on 3D graphene network $\left(\mathrm{MoS}_{2} / 3 \mathrm{DGN}\right)$ and when used as an anode in LIB, this 3D architecture yields a specific capacity of $665 \mathrm{mAhg}^{-1}$ at current density of $0.5 \mathrm{Ag}^{-1}$ after 50 cycles with a good rate performance; even at 4 $\mathrm{Ag}^{-1}$, the reversible capacity is still an impressive 466 $\mathrm{mAhg}^{-1}$ [89]. The deposition of L-TMD NSs on the surface or within the CNT, [42-45] carbon nanotube/fibers [48-50] and amorphous/other form of carbon [92-97] have also been reported as an anode for LIBs. Just like the graphene, the good conductivity of these carbonaceous materials and high specific surface area of L-TMD deposited on them yield enhanced reversible capacity, excellent cyclic stability and improved rate performance compared to their individual counterparts. Kong et al. demonstrated that $\mathrm{MoS}_{2}$ NSs embedded in graphene nanocables $\left(\mathrm{MoS}_{2} @ \mathrm{G}\right)$ could provide high reversible capacity with excellent cyclic stability $\left(1,150 \mathrm{mAhg}^{-1}\right.$ at $0.5 \mathrm{Ag}^{-1}$ after 160 cycles) and extraordinary rate capability (700 $\mathrm{mAhg}^{-1}$ at $\left.10 \mathrm{Ag}^{-1}\right)$. Surprisingly, this hybrid structure delivered high capacity of $900 \mathrm{mAhg}^{-1}$ at $5 \mathrm{Ag}^{-1}$ with long cycle life (700 cycles) [49].

The performance of nanostructured electrodes in LIBs is largely undermined by two key issues which are (1) low conductivity and (2) high volume expansion. Although, a very high initial charge and discharge capacity can be attained by most of the nanostructured electrodes but the rapid capacity fade limits their practical utilization for longer durations. The loss of capacity is a direct result of the large volume expansion during SEI layer formation as a result of lithiation/de-lithiation in the electrode materials. Such limitations could be addressed by tailoring the electrode materials to (1) accommodate the strains and lower the barrier of ion intercalation by synthesizing single layered TMD and (2) composites of TMD with various carbon or other material structures which are flexible in nature and can accommodate strains. Single-layered TMD offers remarkable advantages over multi-layered TMD in terms of large surface area and additional interfacial lithium storage, shortened diffusion pathway to $\mathrm{Li}^{+}$ion, and accommodate the volume change $[48,50]$. Zhu et al. prepared single-layered ultrasmall $\mathrm{MoS}_{2}$ nanoplates uniformly embedded in carbon nanofibers by electrospinning process and this composite showed extraordinary performance for LIBs. Authors further applied TEM modeling to demonstrate that composites were made of single layered and ultrasmall, which were embedded in 
CNFs as shown in Fig. 7a. The composite showed high discharge $\left(1,712 \mathrm{mAhg}^{-1}\right)$ and charge $\left(1,267 \mathrm{mAhg}^{-1}\right)$ capacities at $0.1 \mathrm{Ag}^{-1}$ (Fig. 7b) and outstanding rate performance with specific capacities of 637,548 , and $374 \mathrm{mAhg}^{-1}$ at current density increases from 10, 30, to $50 \mathrm{Ag}^{-1}$ respectively. The cyclic stability was also excellent as the capacity was $661 \mathrm{mAhg}^{-1}$ at $10 \mathrm{Ag}^{-1}$ after 1,000 cycles [48]. Obviously, the enhanced electrochemical performance of these CNFs can be attributed to several factors: (1) Single-layered TMD nanosheets not only provided the insertion and conversion sites for lithium storage, but also numerous interfacial storage sites; (2) The carbon matrix inhibits the restacking of single-layered TMD and aggregation of nanoparticles after cycling; (3) These carbon networks not only solve the volume expansion problem,

(a)

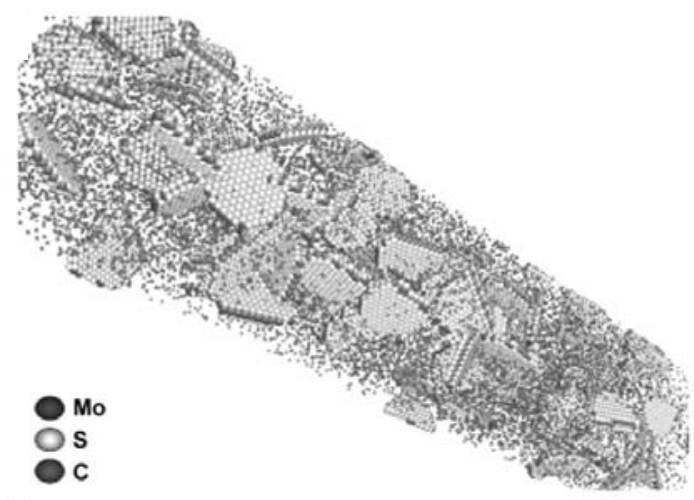

(b)

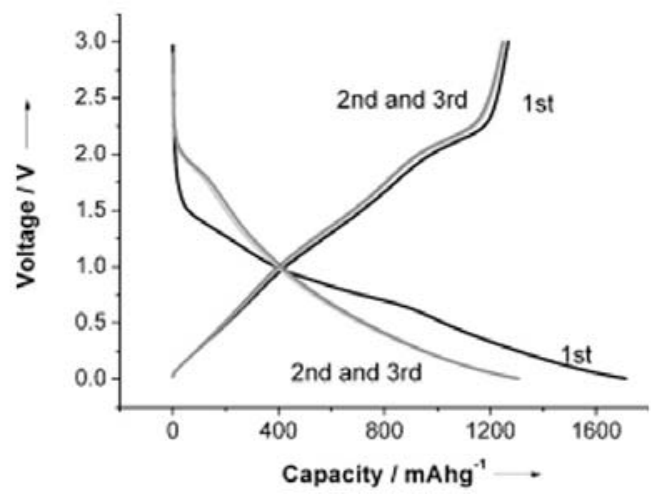

Fig. 7 (a) Schematic illustration based on TEM modeling study. The large black sphere, small black sphere, and white sphere correspond to Mo, C, and S, respectively; (b) Charge and discharge voltage profiles for the first three cycles at 0.1 $\mathrm{Ag}^{-1}$ of the $\mathrm{MoS}_{2}$-carbon fiber composite [48]. Reproduced with permission of John WILEY \& Sons, Inc. but also provide easy access to $\mathrm{Li}^{+}$ion due to 1D character of CNF; (4) Conductivity of carbon networks further increase due to $\mathrm{N}$-doping in CNFs.

Besides carbonaceous materials, composites of noble metal, metal/metal oxide NP-decorated with $\mathrm{MoS}_{2}$ have also been explored for fabricating anodes in LIBs with an enhanced performance [33-38]. The large surface area, elastic and flexible nature of $\mathrm{MoS}_{2} \mathrm{NSs}$ act as an ideal template to inhibit NP aggregation and cushion the volume changes during charging/discharging. The NPs anchored on or in between $\mathrm{MoS}_{2}$ NSs serve as spacer, preventing the restacking of $\mathrm{MoS}_{2}$ during cycling processes and thus improving the capacity retention. Besides, NPs ensure short $\mathrm{Li}^{+}$ion diffusion pathways resulting in superior capacities and rate capabilities [38]. Recently, composites of $1 \mathrm{D} \mathrm{TiO}_{2}$ nanotubes/nanowires/nanobelts [51-53] with $\mathrm{MoS}_{2}$ have also been prepared and considered as alternative anode for LIB with remarkable cycling stability and rate capacity due to their low volume changes during the cycling process and the higher operating voltage of $1.7 \mathrm{~V}$ (compared to $\left.\mathrm{Li}^{+} / \mathrm{Li}\right)$. Nevertheless, $1 \mathrm{D} \quad \mathrm{TiO}_{2}$ and $\mathrm{MoS}_{2}$ nanostructures are interconnected with each other by loose surface contact, which result in self aggregation upon charging/discharging, and hence their performances are still limited. Moreover, 3D porous hollow structures have also been prepared of $\mathrm{TiO}_{2} / \mathrm{MoS}_{2}$ as anode in LIB, in which $\mathrm{MoS}_{2}$ homogenously deposited on $\mathrm{TiO}_{2}$ surface with remarkably improved structural stability and effectively shortened the diffusion path length of both electrons and $\mathrm{Li}^{+}$ions. As a consequence, 3D $\mathrm{TiO}_{2} / \mathrm{MoS}_{2}$ showed a stable cycling performance and superior rate capability [86, 101]. For example, a 3D core shell nanostructure was prepared by coating few layers $\quad\left(<4\right.$ layers) $\quad \mathrm{MoS}_{2}$ on $\mathrm{TiO}_{2} \quad \mathrm{NSs} \quad(3 \mathrm{D}$ FL-MoS $\left.{ }_{2} @ \mathrm{TiO}_{2}\right)$ as an anode for LIB [101]. It exhibited stable cycling performance after 100 cycles with capacity of $713.7 \mathrm{mAhg}^{-1}$ at $0.1 \mathrm{Ag}^{-1}$. 


\subsection{SIBs (Sodium Ion Batteries)}

Although LIBs provide a potential solution to meet the rising demand of global energy for portable electronic devices, EVs and an emerging market for electrical energy storage, we must think about the availability of lithium in the earth's crust. The relative abundance of lithium is still a non-renewable resource and still relatively expensive. On the other hand, $\mathrm{Na}$ (sodium) is a low cost and earth abundant element. In addition, $\mathrm{Al}$ (aluminum) is used as a current collector in SIB, which does not make binary alloy with Na. On this basis, a rechargeable SIB seems to be a more promising alternative to an LIB [102-104]. However, SIBs possess lower theoretical gravimetric and volumetric capacity, because of the poor electrochemical activities compared with LIBs. This is due to 2 intrinsic shortcomings of $\mathrm{Na}$ : firstly, $\mathrm{Na}^{+}$ions are heavier than $\mathrm{Li}^{+}$ions, leading to a sluggish diffusion of $\mathrm{Na}^{+}$ions in bulk of electrode and larger volume expansion, which resulted in low cyclic stability and rate capability, and secondly, the ionization potential of $\mathrm{Na}$ is lower, leading to lower operating voltage, which results in a lowering the gravimetric and volume energy densities. To overcome these deficiencies unique porous crystalline structures with large insertion channels to facilitate sodiation/desodiation have been developed. Recently, a tremendous effort has been made to explore the possibility of using an SIB as an anode. Among them L-TMD NSs based composites have received a lot of attention in recent years. Similar to LIBs, composites of L-TMD with graphene in SIBs have also been investigated as an anode material with or without a binder [55, 62, 69, 83, 105]. For instance, Zhang et al. reported hierarchical $\mathrm{MoSe}_{2} /$ Graphene composite being used as anode in SIB, after 200 cycles, the specific capacity of $430 \mathrm{mAhg}^{-1}$ and $380 \mathrm{mAhg}^{-1}$ at current densities of $0.5 \mathrm{Ag}^{-1}$ and $1 \mathrm{Ag}^{-1}$ were achieved, respectively. This high electrochemical performance was ascribed to synergistic interaction among $\mathrm{MoSe}_{2}$ and GNS [55]. L-TMD composites supported on other carbonaceous materials have also been investigated for application in SIB [46, 48, 95, 98]. Likewise, Zhu et al. fabricated the single-layered $\mathrm{MoS}_{2}$ nanoplates embedded in CNFs and investigated its electrochemical performance. When used as an anode in SIB, it showed an excellent rate capability and cyclic stability. At current densities of 0.1, 0.5, 1, 5, 10, 20, 30, and $50 \mathrm{Ag}^{-1}$, the specific capacities obtained were 854 , $700,623,436,331,224,155$, and $75 \mathrm{mAhg}^{-1}$, respectively. It delivered specific capacities of 484 $\mathrm{mAhg}^{-1}$ and $253 \mathrm{mAhg}^{-1}$, at $1 \mathrm{Ag}^{-1}$ and $10 \mathrm{Ag}^{-1}$ current densities after 100 cycles. This good performance can be attributed to the ultrasmall single-layered $\mathrm{MoS}_{2}$. This is the best reported anode material among all of them for SIB [48]. In another report, PHCSs (porous hollow carbon spheres) were directly decorated by $\mathrm{MoSe}_{2}$ NSs by three steps and tested for electrochemical performance of SIB [95]. At current density of $0.2 \mathrm{Ag}^{-1}$, an outstanding specific capacity $\left(580 \mathrm{mAhg}^{-1}\right)$ was achieved over a prolonged cycle life (100 cycles) and at high current density of $1.5 \mathrm{Ag}^{-1}$, the specific capacity was still maintained to $400 \mathrm{mAhg}^{-1}$. $\mathrm{MoSe}_{2} \mathrm{NSs}$ and PHCS accommodated the stress during charging and discharging. Also PHCS offered the conductivity environment to the composite, while $\mathrm{MoSe}_{2}$ NSs shortened the electronic $\mathrm{Na}^{+}$ion path [95]. In addition, the composites of $\mathrm{MoS}_{2}$ with carbon [98] and $\mathrm{MoSe}_{2}$ with MWCNT [46] have also been demonstrated as anode for SIB with good performance.

\section{Conclusion and Outlook}

Batteries (LIBs/SIBs) are being the future energy storage devices to fulfill the dream by continuously supplying the power to portable electronics and short EVs. Recent advances have shown that L-TMDs are promising materials for LIB and SIB applications, due to their unique physical and chemical properties. When used as an anode in an LIB or SIB, L-TMD NSs are found to be more effective compared to their bulk counterparts because of the higher specific surface area, longer cycle life, higher electronic conductivity and 
shortened path length for ions. Moreover, their performance as measured by the capacity, cyclic stability and rate capability can be further enhanced by hybridization with functional nanomaterials.

In summary, composites of single- and multi-layer TMD have been prepared with OD (noble metal, metal/oxides NPs), 1D (CNT, CNF, $\mathrm{TiO}_{2}$ nanotubes/nanowires/nanobelts), $2 \mathrm{D}$ (graphene, $\mathrm{TiO}_{2}$ nanosheets), 3D (3D graphene, ACF) structures and various other forms of carbon and have shown excellent performance in LIB and SIB. However, most of L-TMD composites with these structures are multilayers instead of single layer, resulting in reduced surface area for lithium and sodium storage. The excellent performance in terms of cycling stability and rate capabilities for LIBs/SIBs have exhibited by single-layered TMD embedded in CNF via electrospinning process. Besides, 3D graphene backbone hybrid porous structures with $\mathrm{MoS}_{2}$ as binder free anode for LIBs have exhibited extraordinary cyclic performance and high rate capacities. Nevertheless, a good performance has been achieved, but some problems are still matter of great concern. The aggregation of TMD during electrode fabrication and degradation of structure during ultralong charging and discharging process (especially at high current density) are inexorable and responsible to their low performance. Moreover, the synthesis of TMD composites with these structures must be scalable before these nanocomposites can be considered for deployment in LIBs/SIBs. To overcome the current challenges, continuous research development is still required to fabricate and explore new L-TMD based composites that yield higher lithium and sodium storage capacity, longer cycle life and higher rate capability.

In future, just like 3D carbon based materials (3D graphene, CNT sponge) offer relatively large surface area, high conductivity and porous structure, 3D macroscopic structures of L-TMD can also be fabricated, and explore their unique properties and a variety of applications. The 3D L-TMDs with large voids can provide the required spaces for fast mass transfer and reversible Li storage for enhanced capacity without any structural degradation. In addition, the yolk-shell structures of L-TMD can be synthesized, which can further provide spaces for reversible $\mathrm{Li}$ and Na storage with enhanced capacity.

\section{References}

[1] Vesborg, P. C. K., and Jaramillo, T. F. 2012. “Addressing the Terawatt Challenge: Scalability in the Supply of Chemical Elements for Renewable Energy." RSC Adv. 2: 7933.

[2] Mahmood, N., and Hou, Y. 2014. "Electrode Nanostructures in Lithium-Based Batteries." Adv. Sci. 1: 140012.

[3] Palacín, M. R. 2009. "Recent Advances in Rechargeable Battery Materials: A Chemist's Perspective." Chem. Soc. Rev. 38: 2565.

[4] Palomares, V., Serras, P., Villaluenga, I., Hueso, K. B., Carretero-González, J., and Rojo, T. 2012. "Na-Ion Batteries, Recent Advances and Present Challenges to Become Low Cost Energy Storage Systems.” Energy Environ. Sci. 5: 5884.

[5] Croguennec, L., and Palacin, M. R. 2015. "Recent Achievements on Inorganic Electrode Materials for Lithium-Ion Batteries." J. Am. Chem. Soc. 137: 3140-56.

[6] Huang, X., Yin, Z., Wu, S., Qi, X., He, Q., Zhang, Q., Yan, Q., Boey, F., and Zhang, H. 2011. "Graphene-Based Materials: Synthesis, Characterization, Properties, and Applications." Small 7: 1876-902.

[7] Geim, A. K., and Novoselov, K. S. 2007. "The Rise of Graphene." Nat. Mater. 6: 183-91.

[8] Pumera, M. 2011. "Graphene-Based Nanomaterials for Energy Storage.” Energy Environ. Sci. 4: 668-74.

[9] Mahmood, N., Zhang, C., Yin, H., and Hou, Y. 2014. "Graphene-Based Nanocomposites for Energy Storage and Conversion in Lithium Batteries, Supercapacitors and Fuel Cells.” J. Mater. Chem. A 2: 15-32.

[10] Srivastava, M., Singh, J., Kuila, T., Layek, R. K., Kim, N. H., and Lee, J. H. 2015. "Recent Advances in Graphene and Its Metal-Oxide Hybrid Nanostructures for Lithium-Ion Batteries." Nanoscale 7: 4820-68.

[11] Huang, X., Zeng, Z., Fan, Z., Liu, J., and Zhang, H. 2012. "Graphene-Based Electrodes." Adv. Mater. 24: 5979-6004.

[12] Zhu, J., Yang, D., Yin, Z., Yan, Q., and Zhang, H. 2014. "Graphene and Graphene-Based Materials for Energy Storage Applications." Small 10: 3480-98.

[13] Luo, B., and Zhi, L. 2015. "Design and Construction of 
Three Dimensional Graphene-Based Composites for Lithium Ion Battery Applications.” Energy Environ. Sci. 8: 456-77.

[14] Raccichini, R., Varzi, A., Passerini, S., and Scrosati, B. 2015. "The Role of Graphene for Electrochemical Energy Storage." Nat. Mater. 14: 271-9.

[15] Han, S., Wu, D., Li, S., Zhang, F., and Feng, X. 2013. "Graphene: A Two-Dimensional Platform for Lithium Storage." Small 9: 1173-87.

[16] Xu, M., Liang, T., Shi, M., and Chen, H. 2013. "Graphene-Like Two-Dimensional Materials." Chem. Rev. 113: 3766-98.

[17] Rao, C. N. R., and Nag, A. 2010. "Inorganic Analogues of Graphene.” Eur. J. Inorg. Chem. 2010: 4244-50.

[18] Pumera, M., Sofer, Z., and Ambrosi, A. 2014. "Layered Transition Metal Dichalcogenides for Electrochemical Energy Generation and Storage." J. Mater. Chem. A 2: 8981.

[19] Chen, D., Chen, W., Ma, L., Ji, G., Chang, K., and Lee, J. Y. 2014. "Graphene-Like Layered Metal Dichalcogenide/Graphene Composites: Synthesis and Applications in Energy Storage and Conversion." Mater. Today 17: 184-93.

[20] Jing, Y., Zhou, Z., Cabrera, C. R., and Chen, Z. 2014. "Graphene, Inorganic Graphene Analogs and Their Composites for Lithium Ion Batteries." J. Mater. Chem. A 2: 12104

[21] Hwang, H., Kim, H., and Cho, J. 2011. " $\mathrm{MoS}_{2}$ Nanoplates Consisting of Disordered Graphene-Like Layers for High Rate Lithium Battery Anode Materials." Nano Lett. 11: 4826-30.

[22] Ko, Y. N., Choi, S. H., Park, S. B., and Kang, Y. C. 2014. "Hierarchical $\mathrm{MoSe}_{2}$ Yolk-Shell Microspheres with Superior Na-Ion Storage Properties." Nanoscale 6: 10511-5.

[23] Koski, K. J., and Cui, Y. 2013. "The New Skinny in Two-Dimensional Nanomaterials." ACS Nano 7: 3739-43.

[24] Gustavsson, F., Svahn, F., Bexell, U., and Jacobson, S. 2013. "Nanoparticle Based and Sputtered $\mathrm{WS}_{2}$ Low-Friction Coatings-Differences and Similarities with Respect to Friction Mechanisms and Tribofilm Formation." Surf. Coat. Technol. 232: 616-26.

[25] André, B., Gustavsson, F., Svahn, F., and Jacobson, S. 2012. "Performance and Tribofilm Formation of a Low-Friction Coating Incorporating Inorganic Fullerene Like Nano-Particles." Surf. Coat. Technol. 206: 2325-9.

[26] Chhowalla, M., Shin, H. S., Eda, G., Li, L.-J., Loh, K. P., and Zhang, H. 2013. "The Chemistry of Two-Dimensional Layered Transition Metal Dichalcogenide Nanosheets." Nat. Chem. 5: 263-75.

[27] Huang, X., Zeng, Z., and Zhang, H. 2013. "Metal Dichalcogenide Nanosheets: Preparation, Properties and
Applications.” Chem. Soc. Rev. 42: 1934-46.

[28] Radisavljevic, B,, Radenovic, A., Brivio, J., Giacometti, V., and Kis, A. 2011. "Single-Layer $\mathrm{MoS}_{2}$ Transistors." Nat. Nanotechnol. 6: 147-50.

[29] Tan, C., and Zhang, H. 2015. "Two-Dimensional Transition Metal Dichalcogenide Nanosheet-Based Composites." Chem. Soc. Rev. 44: 2713-31.

[30] Wang, H., Lan, X., Jiang, D., Zhang, Y., Zhong, H., Zhang, Z., and Jiang, Y. 2015. "Sodium Storage and Transport Properties in Pyrolysis Synthesized $\mathrm{MoSe}_{2}$ Nanoplates for High Performance Sodium-Ion Batteries." J. Power Sources 283: 187-94.

[31] Huang, X., Qi, X., Boey, F., and Zhang, H. 2012. "Graphene-Based Composites." Chem. Soc. Rev. 41: 666-86.

[32] Huang, X., Tan, C., Yin, Z., and Zhang, H. 2014. "25th Anniversary Article: Hybrid Nanostructures Based on Two-Dimensional Nanomaterials." Adv. Mater. 26: 2185-204.

[33] Chen, Y., Song, B., Tang, X., Lu, L., and Xue, J. 2014. "Ultrasmall $\quad \mathrm{Fe}_{3} \mathrm{O}_{4} \quad$ Nanoparticle/ $\mathrm{MoS}_{2} \quad$ Nanosheet Composites with Superior Performances for Lithium Ion Batteries." Small 10: 1536-43.

[34] Pan, L., Zhu, X.-D., Xie, X.-M., and Liu, Y.-T. 2015. "Delicate Ternary Heterostructures Achieved by Hierarchical Co-Assembly of $\mathrm{Ag}$ and $\mathrm{Fe}_{3} \mathrm{O}_{4}$ Nanoparticles on $\mathrm{MoS}_{2}$ Nanosheets: Morphological and Compositional Synergy in Reversible Lithium Storage." J. Mater. Chem. A 3: 2726-33.

[35] Deng, Z., Hu, Y., Ren, D., Lin, S., Jiang, H., and Li, C. 2015. "Reciprocal Hybridization of $\mathrm{MoO}_{2}$ Nanoparticles and Few-Layer $\mathrm{MoS}_{2}$ for Stable Lithium-Ion Batteries." Chem. Commun. 51: 13838-41.

[36] Chen, Y., Lu, J., Wen, S., Lu, L., and Xue, J. 2014. "Synthesis of $\mathrm{SnO}_{2} / \mathrm{MoS}_{2}$ Composites with Different Component Ratios and Their Applications as Lithium Ion Battery Anodes." J. Mater. Chem. A 2: 17857-66.

[37] Li, Q.-Y., Pan, Q.-C., Yang, G.-H., Lin, X.-L., Yan, Z.-X., Wang, H.-Q., and Huang, Y.-G. 2015. "Synthesis of $\mathrm{Sn} / \mathrm{MoS}_{2} / \mathrm{C}$ Composites as High-Performance Anodes for Lithium-Ion Batteries.” J. Mater. Chem. A 3: 20375-81.

[38] Pan, L., Liu, Y. T., Xie, X. M., and Zhu, X. D. 2014. "Coordination-Driven Hierarchical Assembly of Silver Nanoparticles on $\mathrm{MoS}_{2}$ Nanosheets for Improved Lithium Storage." Chem. Asian J. 9: 1519-24.

[39] Shahar, C., Levi, R., Cohen, S. R., and Tenne, R. 2010. "Gold Nanoparticles as Surface Defect Probes for $\mathrm{WS}_{2}$ Nanostructures.” J. Phys. Chem. Lett. 1: 540-3.

[40] Kim, J., Byun, S., Smith, A. J., Yu, J., and Huang, J. 2013. "Enhanced Electrocatalytic Properties of Transition-Metal Dichalcogenides Sheets by Spontaneous Gold Nanoparticle Decoration." J. Phys. Chem. Lett. 4: 
1227-32.

[41] Huang, X., Zeng, Z., Bao, S., Wang, M., Qi, X., Fan, Z., and Zhang, H. 2013. "Solution-Phase Epitaxial Growth of Noble Metal Nanostructures on Dispersible Single-Layer Molybdenum Disulfide Nanosheets." Nat. Commun. 4: 1444.

[42] Ding, S., Chen, J. S., and Lou, X. W. 2011. "Glucose-Assisted Growth of $\mathrm{MoS}_{2}$ Nanosheets on CNT Backbone for Improved Lithium Storage Properties." J. Mater. Chem. A 17: 13142-5.

[43] Park, S. K., Yu, S. H., Woo, S., Quan, B., Lee, D. C., Kim, M. K., Sung, Y. E., and Piao, Y. 2013. "A Simple L-Cysteine-Assisted Method for the Growth of $\mathrm{MoS}_{2}$ Nanosheets on Carbon Nanotubes for High-Performance Lithium Ion Batteries.” Dalton Trans. 42: 2399-405.

[44] Shi, Y., Wang, Y., Wong, J. I., Tan, A. Y., Hsu, C. L., Li, L. J., Lu, Y. C., and Yang, H. Y. 2013. "Self-assembly of Hierarchical $\mathrm{MoS}_{\mathrm{X}} / \mathrm{CNT}$ Nanocomposites $(2<\mathrm{X}<3)$ : Towards High Performance Anode Materials for Lithium Ion Batteries." Sci. Rep. 3: 2169.

[45] Zhai, C., Du, N., Zhang, H., Yu, J., and Yang, D. 2011. "Multiwalled Carbon Nanotubes Anchored with $\mathrm{SnS}_{2}$ Nanosheets as High-Performance Anode Materials of Lithium-Ion Batteries." ACS Appl. Mater. Interfaces 3: 4067-74.

[46] Zhang, Z., Yang, X., Fu, Y. and Du, K. 2015. "Ultrathin Molybdenum Diselenide Nanosheets Anchored on Multi-Walled Carbon Nanotubes as Anode Composites for High Performance Sodium-Ion Batteries." Journal of Power Sources 296: 2-9.

[47] Chang, K., and Chen, W. 2011. "L-Cysteine-Assisted Synthesis of Layered $\mathrm{MoS}_{2} /$ Graphene Composites with Excellent Electrochemical Performances for Lithium Ion Batteries." ACS Nano 5: 4720-8.

[48] Zhu, C., Mu, X., van Aken, P. A., Yu, Y., and Maier, J. 2014. "Single-Layered Ultrasmall Nanoplates of $\mathrm{MoS}_{2}$ Embedded in Carbon Nanofibers with Excellent Electrochemical Performance for Lithium and Sodium Storage." Angew. Chem., Int. Ed. 53: 2152-6.

[49] Kong, D., He, H., Song, Q., Wang, B., Lv, W., Yang, Q.-H., and Zhi, L. 2014. "Rational Design of $\mathrm{MoS}_{2} @$ Graphene Nanocables: Towards High Performance Electrode Materials for Lithium Ion Batteries.” Energy Environ. Sci. 7: 3320-5.

[50] Yu, S., Jung, J. W., and Kim, I. D. 2015. "Single Layers of $\mathrm{WS}_{2}$ Nanoplates Embedded in Nitrogen-Doped Carbon Nanofibers as Anode Materials for Lithium-Ion Batteries." Nanoscale 7: 11945-50.

[51] Xu, X., Fan, Z., Ding, S., Yu, D., and Du, Y. 2014. "Fabrication of $\mathrm{MoS}_{2}$ Nanosheet@ $\mathrm{TiO}_{2}$ Nanotube Hybrid Nanostructures for Lithium Storage." Nanoscale 6: 5245-50.
[52] Li, X., Li, W., Li, M., Cui, P., Chen, D., Gengenbach, T., Chu, L., Liu, H., and Song, G. 2015. "Glucose-Assisted Synthesis of the Hierarchical $\mathrm{TiO}_{2}$ Nanowire@ $\mathrm{MoS}_{2}$ Nanosheet Nanocomposite and Its Synergistic Lithium Storage Performance.” J. Mater. Chem. A 3: 2762-9.

[53] Mao, M., Mei, L., Guo, D., Wu, L., Zhang, D., Li, Q., and Wang, T. 2014. "High Electrochemical Performance Based on the $\mathrm{TiO}_{2}$ Nanobelt@Few-Layered $\mathrm{MoS}_{2}$ Structure for Lithium-Ion Batteries." Nanoscale 6: 12350-3.

[54] Chang, K., and Chen, W. 2011. "In Situ Synthesis of $\mathrm{MoS}_{2} /$ Graphene Nanosheet Composites with Extraordinarily High Electrochemical Performance for Lithium Ion Batteries.” Chem. Commun. 47: 4252-4.

[55] Zhang, Z., Fu, Y., Yang, X., Qu, Y., and Zhang, Z. 2015. "Hierarchical $\mathrm{MoSe}_{2}$ Nanosheets/Reduced Graphene Oxide Composites as Anodes for Lithium-Ion and Sodium-Ion Batteries with Enhanced Electrochemical Performance." ChemNanoMat 1: 409-14.

[56] Wang, Z., Chen, T., Chen, W., Chang, K., Ma, L., Huang, G., Chen, D., and Lee, J. Y. 2013. "CTAB-Assisted Synthesis of Single-Layer $\mathrm{MoS}_{2}-$ Graphene Composites as Anode Materials of Li-Ion Batteries." J. Mater. Chem. A 1: 2202-10.

[57] Wang, Z., Ma, L., Chen, W., Huang, G., Chen, D., Wang, L., and Lee, J. Y. 2013. "Facile Synthesis of $\mathrm{MoS}_{2} /$ Graphene Composites: Effects of Different Cationic Surfactants on Microstructures and Electrochemical Properties of Reversible Lithium Storage." RSC Adv. 3: 21675.

[58] Huang, G., Chen, T., Chen, W., Wang, Z., Chang, K., Ma, L., Huang, F., Chen, D., and Lee, J. Y. 2013. "Graphene-Like $\mathrm{MoS}_{2}$ Graphene Composites: Cationic Surfactant-Assisted Hydrothermal Synthesis and Electrochemical Reversible Storage of Lithium." Small 9: 3693-703.

[59] Ma, L., Zhou, X., Xu, L., Xu, X., Zhang, L., and Chen, W. 2015. "Ultrathin Few-Layered Molybdenum Selenide/Graphene Hybrid with Superior Electrochemical Li-Storage Performance." J. Power Sources 285: 274-80.

[60] Chang, K., and Chen, W. 2011. "Single-Layer $\mathrm{MoS}_{2} /$ Graphene Dispersed in Amorphous Carbon: Towards High Electrochemical Performances in Rechargeable Lithium Ion Batteries.” J. Mater. Chem. 21: 17175.

[61] Chang, K., Geng, D., Li, X., Yang, J., Tang, Y., Cai, M., Li, R., and Sun, X. 2013. "Ultrathin $\mathrm{MoS}_{2} /$ Nitrogen-Doped Graphene Nanosheets with Highly Reversible Lithium Storage." Adv. Energy Mater. 3: 839-44.

[62] Sahu, T. S., and Mitra, S. 2015. "Exfoliated $\mathrm{MoS}_{2}$ Sheets and Reduced Graphene Oxide-an Excellent and Fast Anode for Sodium-Ion Battery.” Sci. Rep. 5: 12571. 
[63] Jing, Y., Ortiz-Quiles, E. O., Cabrera, C. R., Chen, Z., and Zhou, Z. 2014. "Layer-by-Layer Hybrids of $\mathrm{MoS}_{2}$ and Reduced Graphene Oxide for Lithium Ion Batteries." Electrochim. Acta 147: 392-400.

[64] Chen, P., Su, Y., Liu, H., and Wang, Y. 2013. "Interconnected Tin Disulfide Nanosheets Grown on Graphene for Li-Ion Storage and Photocatalytic Applications.” ACS Appl. Mater. Interfaces 5: 12073-82.

[65] Zhuo, L., Wu, Y., Wang, L., Yu, Y., Zhang, X., and Zhao, F. 2012. "One-Step Hydrothermal Synthesis of $\mathrm{SnS}_{2} /$ Graphene Composites as Anode Material for Highly Efficient Rechargeable Lithium Ion Batteries.” RSC Adv. 2: 5084 .

[66] Chang, K., Wang, Z., Huang, G., Li, H., Chen, W., and Lee, J. Y. 2012. "Few-Layer $\mathrm{SnS}_{2} /$ Graphene Hybrid with Exceptional Electrochemical Performance as Lithium-Ion Battery Anode." J. Power Sources 201: 259-66.

[67] Jiang, Z., Wang, C., Du, G., Zhong, Y. J., and Jiang, J. Z. 2012. "In Situ Synthesis of $\mathrm{SnS}_{2} @$ Graphene Nanocomposites for Rechargeable Lithium Batteries." J. Mater. Chem. 22: 9494.

[68] Choi, J., Jin, J., Jung, I. G., Kim, J. M., Kim, H. J., and Son, S. U. 2011. " $\mathrm{SnSe}_{2}$ Nanoplate-Graphene Composites as Anode Materials for Lithium Ion Batteries." Chem. Commun. 47: 5241-3.

[69] Qu, B., Ma, C., Ji, G., Xu, C., Xu, J., Meng, Y. S., Wang, T., and Lee, J. Y. 2014. "Layered $\mathrm{Sns}_{2}$-Reduced Graphene Oxide Composite - a High-Capacity, High-Rate, and Long-Cycle Life Sodium-Ion Battery Anode Material." Adv. Mater. 26: 3854-9.

[70] Luo, B., Fang, Y., Wang, B., Zhou, J., Song, H., and Zhi, L. 2012. "Two Dimensional Graphene- $\mathrm{SnS}_{2}$ Hybrids with Superior Rate Capability for Lithium Ion Storage.” Energy Environ. Sci. 5: 5226-30.

[71] Shiva, K., Ramakrishna Matte, H. S. S., Rajendra, H. B., Bhattacharyya, A. J., and Rao, C. N. R. 2013. "Employing Synergistic Interactions between Few-Layer $\mathrm{WS}_{2}$ and Reduced Graphene Oxide to Improve Lithium Storage, Cyclability and Rate Capability of Li-Ion Batteries." Nano Energy 2: 787-93.

[72] Xu, X., Rout, C. S., Yang, J., Cao, R., Oh, P., Shin, H. S., and Cho, J. 2013. "Freeze-Dried $\mathrm{WS}_{2}$ Composites with Low Content of Graphene as High-Rate Lithium Storage Materials." J. Mater. Chem. A 1: 14548.

[73] Chen, D., Ji, G., Ding, B., Ma, Y., Qu, B., Chen, W., and Lee, J. Y. 2013. "In Situ Nitrogenated Graphene-Few-Layer $\mathrm{WS}_{2}$ Composites for Fast and Reversible Li+ Storage." Nanoscale 5: 7890-6.

[74] Li, H., Yu, K., Fu, H., Guo, B., Lei, X., and Zhu, Z. 2015. "Multi-Slice Nanostructured WS $@$ @RGO with Enhanced Li-Ion Battery Performance and a Comprehensive Mechanistic Investigation.” Phys. Chem. Chem. Phys. 17:
29824-33.

[75] Gong, Y., Yang, S., Zhan, L., Ma, L., Vajtai, R., and Ajayan, P. M. 2014. "A Bottom-up Approach to Build 3D Architectures from Nanosheets for Superior Lithium Storage." Adv. Funct. Mater. 24: 125-30.

[76] Gong, Y., Yang, S., Liu, Z., Ma, L., Vajtai, R., and Ajayan, P. M. 2013. "Graphene-Network-Backboned Architectures for High-Performance Lithium Storage." Adv. Mater. 25: 3979-84.

[77] Lingappan, N., Van, N. H., Lee, S., and Kang, D. J. 2015. "Growth of Three Dimensional Flower-Like Molybdenum Disulfide Hierarchical Structures on Graphene/Carbon anotube Network: An Advanced Heterostructure for Energy Storage Devices.” J. Power Sources 280: 39-46.

[78] Yao, J., Liu, B., Ozden, S., Wu, J., Yang, S., Rodrigues, M.-T. F., Kalaga, K., Dong, P., Xiao, P., Zhang, Y., Vajtai, R., and Ajayan, P. M. 2015. "3D Nanostructured Molybdenum Diselenide/Graphene Foam as Anodes for Long-Cycle Life Lithium-Ion Batteries." Electrochim. Acta 176: 103-11.

[79] Miao, Y. E., Huang, Y., Zhang, L., Fan, W., Lai, F., and Liu, T. 2015. "Electrospun Porous Carbon Nanofiber@ $\mathrm{MoS}_{2}$ Core/Sheath Fiber Membranes as Highly Flexible and Binder-Free Anodes for Lithium-Ion Batteries." Nanoscale 7: 11093-101.

[80] Jiang, X., Yang, X., Zhu, Y., Shen, J., Fan, K., and Li, C. 2013. "In Situ Assembly of Graphene Sheets-Supported $\mathrm{SnS}_{2}$ Nanoplates into 3d Macroporous Aerogels for High-Performance Lithium Ion Batteries.” J. Power Sources 237: 178-86.

[81] Tang, H., Qi, X., Han, W., Ren, L., Liu, Y., Wang, X., and Zhong, J. 2015. " $\mathrm{SnS}_{2}$ Nanoplates Embedded in 3D Interconnected Graphene Network as Anode Material with Superior Lithium Storage Performance." Appl. Surf. Sci. 355: 7-13.

[82] Huang, G., Liu, H., Wang, S., Yang, X., Liu, B., Chen, H., and $\mathrm{Xu}, \mathrm{M}$. 2015. "Hierarchical Architecture of $\mathrm{WS}_{2}$ Nanosheets on Graphene Frameworks with Enhanced Electrochemical Properties for Lithium Storage and Hydrogen Evolution.” J. Mater. Chem. A 3: 24128-38.

[83] Su, D., Dou, S., and Wang, G. 2014. “WS $@$ Graphene Nanocomposites as Anode Materials for Na-Ion Batteries with Enhanced Electrochemical Performances." Chem. Commun. 50: 4192-5.

[84] Liu, Y., Wang, W., Wang, Y., and Peng, X. 2014. "Homogeneously Assembling Like-Charged $\mathrm{WS}_{2}$ and GO Nanosheets Lamellar Composite Films by Filtration for Highly Efficient Lithium Ion Batteries." Nano Energy 7: 25-32.

[85] Huang, Y., Wu, D., Wang, J., Han, S., Lv, L., Zhang, F., and Feng, X. 2014. "Amphiphilic Polymer Promoted Assembly of Macroporous Graphene/ $\mathrm{SnO}_{2}$ Frameworks 
with Tunable Porosity for High-Performance Lithium Storage." Small 10: 2226-32.

[86] Guo, B., Yu, K., Fu, H., Hua, Q., Qi, R., Li, H., Song, H., Guo, S., and Zhu, Z. 2015. "Firework-Shaped $\mathrm{TiO}_{2}$ Microspheres Embedded with Few-Layer $\mathrm{MoS}_{2}$ as an Anode Material for Excellent Performance Lithium-Ion Batteries." J. Mater. Chem. A 3: 6392-401.

[87] Chen, Z., Ren, W., Gao, L., Liu, B., Pei, S., and Cheng, H.-M. 2011. "Three-Dimensional Flexible and Conductive Interconnected Graphene Networks Grown by Chemical Vapour Deposition.” Nat. Mater. 10: 424-8.

[88] Cao, X., Yin, Z., and Zhang, H. 2014. "Three-Dimensional Graphene Materials: Preparation, Structures and Application in Supercapacitors." Energy Environ. Sci. 7: 1850-65.

[89] Cao, X., Shi, Y., Shi, W., Rui, X., Yan, Q., Kong, J., and Zhang, H. 2013. "Preparation of $\mathrm{MoS}_{2}$-Coated Three-Dimensional Graphene Networks for High-Performance Anode Material in Lithium-Ion Batteries." Small 9: 3433-8.

[90] Wang, C., Wan, W., Huang, Y., Chen, J., Zhou, H. H., and Zhang, X. X. 2014. "Hierarchical $\mathrm{MoS}_{2}$ Nanosheet/Active Carbon Fiber Cloth as a Binder-Free and Free-Standing Anode for Lithium-Ion Batteries." Nanoscale 6: 5351-8.

[91] Liu, K. K., Zhang, W., Lee, Y. H., Lin, Y. C., Chang, M. T., Su, C. Y., Chang, C. S., Li, H., Shi, Y., Zhang, H., Lai, C. S., and Li, L. J. 2012. "Growth of Large-Area and Highly Crystalline $\mathrm{MoS}_{2}$ Thin Layers on Insulating Substrates." Nano Lett. 12: 1538-44.

[92] Chang, K., Chen, W., Ma, L., Li, H., Li, H., Huang, F., Xu, Z., Zhang, Q., and Lee, J.-Y. 2011. "Graphene-Like $\mathrm{MoS}_{2} /$ Amorphous Carbon Composites with High Capacity and Excellent Stability as Anode Materials for Lithium Ion Batteries.” J. Mater. Chem. 21: 6251.

[93] Das, S. K., Mallavajula, R., Jayaprakash, N., and Archer, L. A. 2012. "Self-assembled $\mathrm{MoS}_{2}$-Carbon Nanostructures: Influence of Nanostructuring and Carbon on Lithium Battery Performance.” J. Mater. Chem. 22: 12988.

[94] Yu, X. Y., Hu, H., Wang, Y., Chen, H., and Lou, X. W. 2015. "Ultrathin $\mathrm{MoS}_{2}$ Nanosheets Supported on N-Doped Carbon Nanoboxes with Enhanced Lithium Storage and Electrocatalytic Properties." Angew. Chem.,
Int. Ed. 54: 7395-8.

[95] Yang, X., Zhang, Z., Fu, Y., and Li, Q. 2015. "Porous Hollow Carbon Spheres Decorated with Molybdenum Diselenide Nanosheets as Anodes for Highly Reversible Lithium and Sodium Storage." Nanoscale 7: 10198-203.

[96] Liu, Y., Zhu, M., and Chen, D. 2015. "Sheet-Like $\mathrm{MoSe}_{2} / \mathrm{C}$ Composites with Enhanced Li-Ion Storage Properties." J. Mater. Chem. A 3: 11857-62.

[97] Hu, S., Chen, W., Zhou, J., Yin, F., Uchaker, E., Zhang, Q., and Cao, G. 2014. "Preparation of Carbon Coated $\mathrm{MoS}_{2}$ Flower-Like Nanostructure with Self-assembled Nanosheets as High-Performance Lithium-Ion Battery Anodes." J. Mater. Chem. A 2: 7862.

[98] Wang, Y.-X., Seng, K. H., Chou, S.-L., Wang, J.-Z., Guo, Z., Wexler, D., Liu, H.-K., and Dou, S.-X. 2014. "Reversible Sodium Storage via Conversion Reaction of a $\mathrm{MoS}_{2}$-C Composite." Chem. Commun. 50: 10730-3.

[99] Bruce, P. G., Scrosati, B., and Tarascon, J. M. 2008. "Nanomaterials for Rechargeable Lithium Batteries." Angew. Chem., Int. Ed. 47: 2930-46.

[100] Xiao, J., Choi, D., Cosimbescu, L., Koech, P., Liu, J., and Lemmon, J. P. 2010. "Exfoliated $\mathrm{MoS}_{2}$ Nanocomposite as an Anode Material for Lithium Ion Batteries." Chem. Mater. 22: 4522-4.

[101] Chen, B., Zhao, N., Guo, L., He, F., Shi, C., He, C., Li, J., and Liu, E. 2015. "Facile Synthesis of 3D Few-Layered $\mathrm{MoS}_{2}$ Coated $\mathrm{TiO}_{2}$ Nanosheet Core-Shell Nanostructures for Stable and High-Performance Lithium-Ion Batteries." Nanoscale 7: 12895-905.

[102] Yabuuchi, N., Kubota, K., Dahbi, M., and Komaba, S. 2014. "Research Development on Sodium-Ion Batteries." Chem. Rev. 114: 11636-82.

[103] Sawicki, M., and Shaw, L. L. 2015. "Advances and Challenges of Sodium Ion Batteries as Post Lithium Ion Batteries." RSC Adv. 5: 53129-54.

[104] Kundu, D., Talaie, E., Duffort, V., and Nazar, L. F. 2015. "The Emerging Chemistry of Sodium Ion Batteries for Electrochemical Energy Storage.” Angew. Chem., Int. Ed. 54: 3431-48.

[105] David, L., Bhandavat, R., and Singh, G. 2014. " $\mathrm{MoS}_{2} /$ Graphene Composite Paper for Sodium-Ion Battery Electrodes." ACS Nano 8: 1759-70. 